\title{
Workbench Film Thickness Detection Based on Laser Sensor
}

\author{
Mo-Yun LIU, Han-Bing TANG*, Ma-Chao JING, and Zhen ZHOU
}

\author{
School of Mechanical Engineering, Hubei University of Technology, Wuhan, Hubei, China
}

*E-mail: tanghbhgd@163.com

www.hbut.edu.cn

Keywords: Laser displacement sensor, Oil film thickness, Static workbench.

\begin{abstract}
For large heavy-duty rotary table, in order to improve the machining precision of the workbench in practical work, the workbench is usually set above the oil film with stable thickness. Therefore, the accurate measurement of the oil film thickness of the workbench hydrostatic guide rail is very important for improving the accuracy of the workbench. So a high-precision laser displacement sensor measurement method is proposed to monitor the oil film thickness changes of the guide rail in real-time, and the experimental results show that the introduction of laser sensor in the oil film measurement of worktable has obvious advantages, it also provides a feasible experimental method for practical measurement.
\end{abstract}

\section{Introduction}

Static workbench is one of the important components of vertical CNC machine tool. In order to meet the requirements of high precision, it is necessary to ensure the stability of the oil film thickness between the bottom of the table and the hydrostatic guide rail under different loads. Therefore, the accurate measurement of the oil film thickness of the workbench hydrostatic guide rail is very important for improving the machining quality and the precision of machine tool. In order to solve the problem of oil film thickness detection, in recent years, many experts and scholars at home and abroad have conducted in-depth study.

Liu[1] has comprehensively reviewed a variety of measurement methods, such as resistance method, discharge voltage method, capacitance method, eddy current method, optical interferometry, X-ray method, reluctance method, strain gauge method, ultrasonic method and so on[2-4]. Dhar[5] proposed a method to measure lubrication oil film thickness between the plunger ring and pad lining contact surface by capacitance microsensor method. Luo[6] puts forward the method that using grating microsensor to measure the variation of workbench displacement to indirectly measure the oil film thickness. However, these methods must modify part of the structure of the measured object, and there are many shortcomings, such as difficult to set, measurement accuracy is not high, easy to short-circuit and susceptible to electromagnetic interference, in-situ measurement is limited, higher requirements of experimental conditions.

Different from the above measuring methods, laser displacement sensor has been increasingly applied in practical scientific research project due to its advantages of high precision, good real-time, no damage and so on. This paper proposed a method for measuring the thickness of oil film based on laser sensor, which have not limitations on the material and structure of measured object, and it is more suitable to the measurement of lubricant oil film thickness in industrial field. At the same time the sensor uses high-speed data acquisition and real-time processing system, can achieve rapid and continuous displacement measurement. Therefore, it is significant to research a new method for the measurement of lubricant film thickness of rotary table, which is applied to the actual table detection and fault diagnosis.

\section{The Principle of Laser Sensor Detection}

At present, the method of measuring the oil film thickness of the large rotary table is usually using the micrometer with low accuracy, the capacitance method which is easy to short circuit and the 
grating micro sensor with slow response, and there is no real breakthrough in high speed displacement measurement.

\section{Laser Displacement Sensor}

The US MT3 laser displacement sensor adopts the adaptive control method to adjust the intensity of the emitted laser light, and automatically adjusts the measurement frequency according to the measurement object, thus adapting to various measured objects and external environmental conditions. Laser displacement measurement system is composed of laser diode, optical system, linear CCD sensor, digital signal processor and external interface, etc. [7]. The sensor uses analog triangulation technology to detect the displacement and thickness. The position of the reflected light on the probe changes with the position of the target. The displacement of the object can be measured by detecting the change. Laser sensor is not subject to surface material, color and emission of light effects, and has a wide range of applications. The visible laser light spot makes laser probe operation and positioning easier. Text numerical LCD display can display test results, set parameters and other control parameters. Cut Time function can ignore the hole and the gap of the instantaneous measurement of impact. Changeable laser head do not need to correct, has a great anti-interference ability, and a good performance of high-precision, long-distance and fast sampling. It can measure the change of oil film thickness at high speed and high accuracy [8].

\section{The Measurement Principle of Laser Displacement Sensor}

Laser displacement sensor measurement principle was shown in Fig. 1.

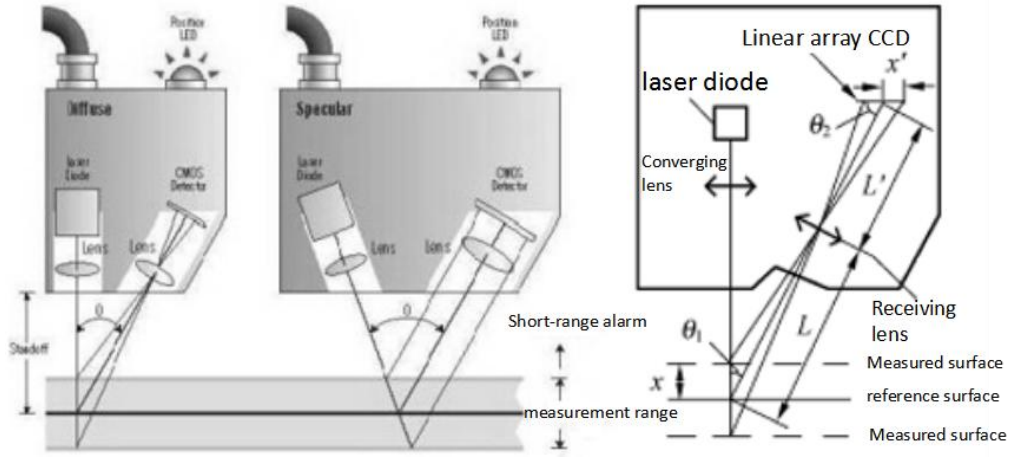

Figure 1. Measurement principle of laser displacement sensor

The laser beam emitted from the laser diode is focused by the focusing lens and is incident on the surface of the object under test, when the measured surface have displacement in this direction due to movement of the object, the receiving lens receives the scattered light at the incident light spot on the surface of the measured object, which are imaged at different positions on the CCD sensitive surface. According to the spot on CCD, the sensor using its own algorithm to measure the displacement value of the object. In the Fig.1, L is the distance from the intersection of the optical axis of the laser beam and the receiving optical axis to the front surface of the receiving lens[7].

\section{The Experimental Principle}

Research object is YK312xx numerical control hobbing machine. In workbench hydrostatic guide rail uses static pressure unloading technology, choosing fan-shaped hydrostatic pad as a support rail, long oil pump for the oil chamber, MT3 high-speed laser sensor as a detection element. In order to verify the feasibility of using laser displacement sensing technology to detect oil film thickness of hydrostatic table, a simple test bed for oil film thickness measurement and a measurement and analysis system should be set up.

Principle of the process shown in Fig. 2 


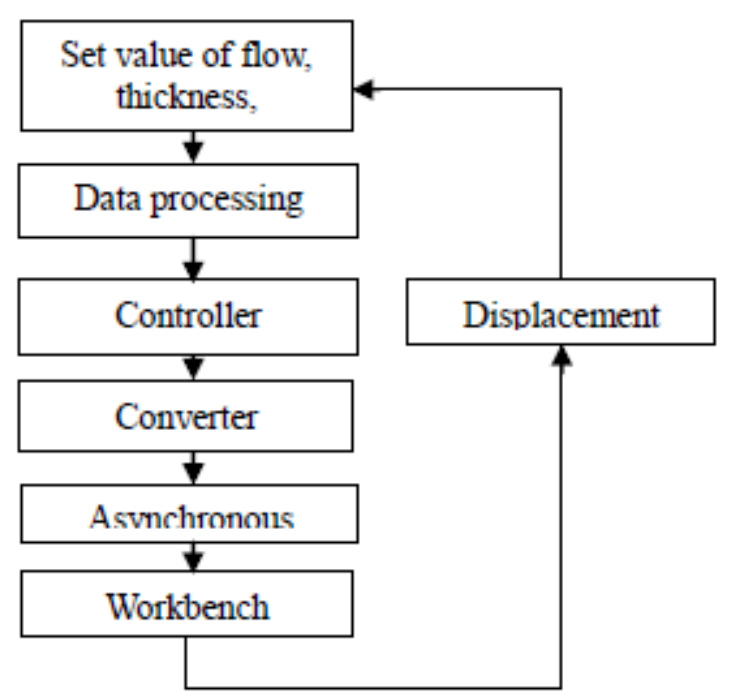

Figure 2. Working principle flow chart

The whole control system has the advantages of high precision, fast, no contact, the working principle is that the target oil film thickness is inputted from the numerical control device panel, and the numerical control system outputs the control signal of the asynchronous motor through the control algorithm set in the system. After the motor speed is stabilized, the pressure and the load of the hydraulic oil are matched, and the numerical control system will process and analyze the oil film thickness data signal gathered form sensor in real-time, and according to the analysis results, it will automatically determine whether it is necessary to adjust the motor speed again to adjust the oil film thickness. Similarly, if add the external load, the sensor will detect changes in film thickness, and pass the feedback to the control system, repeat the working principle of the steps so as to achieve the purpose of automatic adjustment of film thickness.

Theoretically, it is necessary to measure the thickness of the oil film between the upper surface of the sector and the lower surface of the table, but the oil film thickness between the lubricating pad and the table is thin, it is inconvenient to install and remove the sensor, So change the measurement method in the actual experimental process, mount the laser probe fixedly above the table, This method requires a small distance between the sensor head and the surface of the table, and requires the position of the reference standard to be detected. The change of the distance between the workbench and the laser probe can indirectly reflect the change of the oil film thickness.

\section{Experimental Design and Results Analyze}

In order to verify the feasibility of the workbench laser sensor detection system, doing preliminary test in simple equipment platform which has been built. At first, we input target oil film thickness, maximum flow, limit temperature and other set point in numerical control system, and in the meantime data processing system unit is receiving the signals from recess pressure, the temperature of lubricant and detection feedback system of oil film thickness, obtaining corresponding frequency after comparing them with each other, and then transport to the controller. The alternating-current transducer receive the output signals of controller to actuate multi-head pump AC variable-frequency electric motor, then control the flow of multi-head pump oil chamber. Start hydraulic system and numerical control system, and set the maximum allowed value of oil film thickness at the same time. When it is greater than the value, early warning signal corresponding to laser displacement sensor will be fed back to control system, which makes workbench alarm or stop automatically. Under this condition, the workbench is loaded to test the change of displacement between laser probe and workbench under different external load, and then calculate corresponding change of oil film thickness based on it.

This experiment was done in YK312xx CNC gear hobbing machine developed by a company, using American MT3 laser displacement sensor, whose installation reference distance is $25 \mathrm{mmm}$, 
measuring distance is $2 \mathrm{~mm}$, and measurement accuracy is $0.12 \mathrm{um}$. During the experiment, using the same lubricant input the same flow lubricant under different loading conditions, and records the changing data of oil film thickness as detected by a laser probe at different flow rates.

To deal with the data detected by sensor, get the relationship between the oil film thickness and the flow of multi-head pump when loading is same. Table 1 shows relevant data.

Table 1. Table of the relationship between oil film thickness and oil supply flow under the same load

\begin{tabular}{cccccc}
\hline \multicolumn{6}{c}{ Oil film thickness under different load/mm } \\
\hline Load/N & Flow1 & Flow2 & Flow3 & Flow4 & Flow5 \\
\hline 0(self-weight) & 0 & 0.0182 & 0.0322 & 0.0412 & 0.0494 \\
50000 & 0 & 0.0148 & 0.0221 & 0.0279 & 0.0326 \\
100000 & 0 & 0.0117 & 0.0184 & 0.0239 & 0.0283 \\
150000 & 0 & 0.0096 & 0.0167 & 0.0208 & 0.024 \\
\hline
\end{tabular}

According to analyze results of above data, Under the same load, the relationship between the oil film thickness and amount of fuel is shown in Fig. 3, At the same time can get the relationship between the oil film thickness and different loading when flow is same, as shown in Fig. 4, take flow 3 as an example.

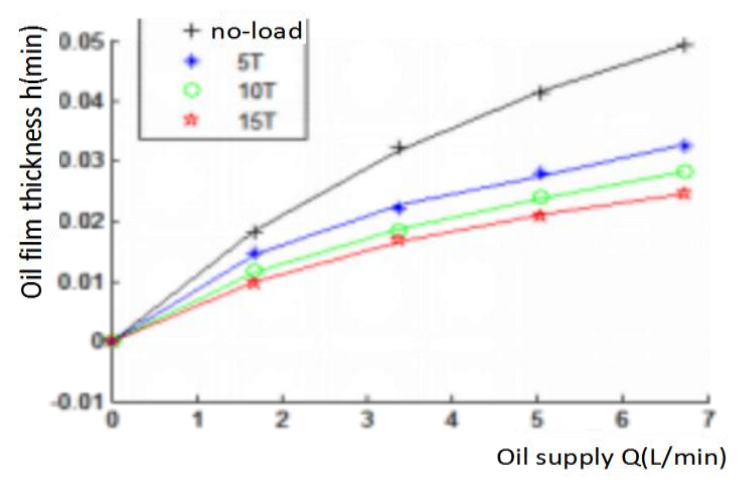

Figure 3 Relationship between oil film thickness and oil supply

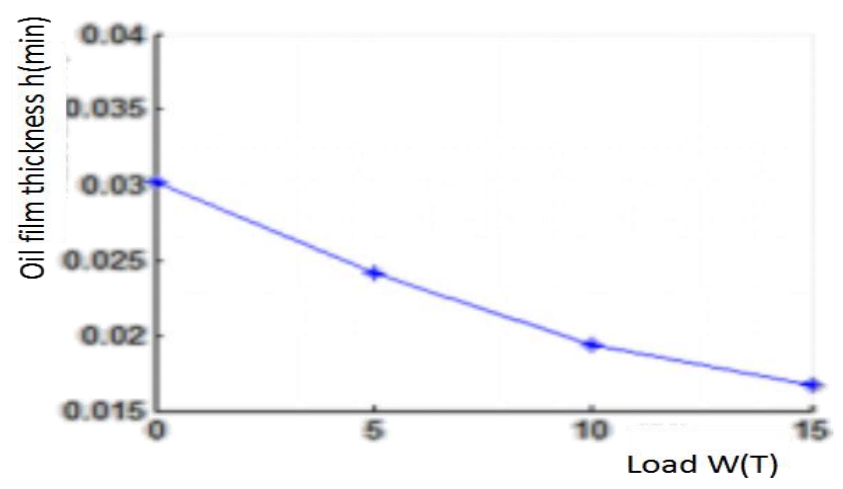

Figure 4 Relationship oil film thickness and load

From Fig. 3 and Table 1 available in the same load, the oil film thickness of the table increases with the oil supply. This is because when the load of the table is constant, the gap between the table and the base will increase with the increase of the oil supply. It can be seen from Figure 4 that with the increasing of the load, the oil film thickness will decrease under the same oil supply. This is because: the load force increases, the same amount of fuel oil table under the film thickness will be reduced. 


\section{Conclusions}

This paper introduces the measurement principle of high-speed laser displacement sensor, and a control scheme of oil film thickness real-time detection based on laser sensor technology is established and a simple oil film thickness test is designed. The feasibility of using laser displacement sensor to measure the oil film thickness of the worktable is confirmed by the experiment, the relationship between oil supply, load and oil film thickness of hydrostatic pad is obtained by measuring the displacement of hydrostatic pad under different load and oil supply.

\section{References}

1. WJH Okkerse, SPP Ottengraf, B Osinga-Kuipers, Biotec Bioeng 6, 619-629 (2000)

2. H J Weimar, U. Spicher, ASME Conference 349-359 (2003)

3. K. Kc, B. Oh S, H. Kim S, et al. MEAS SCI TECHNOL 10, 1683-1688 (2001)

4. Y Qin, LUB ENG 4, 60-64 (2006).

5. A. Dhar, AK. Agarwal, V. Saxena, M Sensors and Actuators, A: Physical, 1, 7-15 (2009)

6. J Han, H Xia, L Xia. G CJME, 21, 2891-2895 (2014)

7. J Chen, X Wang, et al. O\&PE 4, 611-616 (2008)

8. W ShaoLi, X WanLi, M ShuGuang, et al. JMS (2015) 